\title{
A SURDEZ COMO DIFERENÇA: NOTAS DE PESQUISA
}

\author{
DEAFNESS AS DIFFERENCE: RESEARCH NOTES
}

\author{
Paulo Roberto Tonani do Patrocínio, Ddináh Maria dos Santos ${ }^{1}$ \\ 'Universidade Federal do Rio de Janeiro (UFRJ), Rio de Janeiro, RJ, Brasil \\ paulotonani@gmail.com;dynwdina@hotmail.com
}

Recebido em: 25 abr. 2018

Aceito em: 7 de jun. 2018

Resumo: $O$ presente ensaio tem como principal objetivo a análise das contribuições críticas e teóricas dos Estudos Culturais acerca da compreensão da surdez enquanto diferença, rompendo com a ideia de deficiência que orientava as leituras da surdez e do sujeito surdo. Parte-se do pressuposto que as representações da surdez devem ser lidas enquanto reflexo e efeito primeiro de reflexões e configurações discursivas de saber-poder que resultam em representações que transitam entre um modelo médico terapêutico - baseado em uma perspectiva que nomeia o sujeito surdo enquanto deficiente e lança mão de estratégias e recursos clínicos que almejam a sua reabilitação - e um modelo socioantropológico - fundado em uma perspectiva culturalista inspirada nos Estudos Culturais que nomeia a surdez enquanto uma diferença que constitui uma cultura própria, a Cultura Surda. A presente análise busca identificar os principais trabalhos e pesquisas produzidas no âmbito acadêmico brasileiro que promovem uma compreensão da comunidade surda enquanto um grupo minoritário que instaura um elemento de distinção na cultura hegemônica e funda um campo de investigação próprio denominado Estudos Surdos, uma espécie de apropriação do arcabouço teórico dos Estudos Culturais para pensar a surdez e suas representações e autorrepresentações.

Palavras-chave: Estudos Culturais; Diferença; Surdez; Identidade Surda.

\begin{abstract}
The main aim of this essay is to analyze the critical and theoretical contributions from Cultural Studies to understanding of deafness as difference, breaking with the idea of disability which guided the readings of deafness and of the deaf subject. It is assumed that the representations of deafness should be read as foremost reflection and effect of discursive reflection and configurations of power-knowledge which result in representations that move between a therapeutic medical model - based on a perspective that names the deaf subject as deficient and makes use of strategies and clinical resources which aim at his rehabilitation - and a socio-anthropological model - grounded in a culturalist perspective inspired by the Cultural Studies that names deafness as an difference which constitutes its own culture, the Deaf Culture. This examination attempts to identify the main works and researches produced in the Brazilian academic realm that promote an understanding of the deaf community as a minority group that establishes a distinguishing element in hegemonic culture as well as its own investigation field named Deaf Studies, a kind of appropriation of the theoretical framework of Cultural Studies to examine deafness, its representations and self-representations.
\end{abstract}

Keywords: Cultural Studies; Difference; Deaf; Deaf Identity. 
O presente artigo apresenta alguns dos principais resultados obtidos no âmbito de uma pesquisa de natureza teórica que tem como objetivo analisar as contribuições dos Estudos Culturais acerca dos discursos da diferença, buscando analisar de que forma este debate influenciou a emergência de uma compreensão da surdez em uma perspectiva socioantropológica que a concebe enquanto uma diferença. Em síntese, a investigação tem como objetivo identificar os principais trabalhos e pesquisas produzidas no âmbito acadêmico brasileiro que promovem uma compreensão da comunidade surda enquanto um grupo minoritário que instaura um elemento de distinção na cultura hegemônica.

Parte-se do pressuposto que a emergência desta concepção da surdez e do sujeito surdo representa uma mudança epistemológica que rompe com a concepção clínica que regia representações baseadas em um conceito de deficiência. Fundada em um discurso de acento pós-estruturalista e inspirada nos pressupostos teóricos dos Estudos Culturais, a visão socioantropológica da surdez aciona o conceito de diferença como elemento formador de uma nova episteme para tratamento do sujeito surdo e da surdez. Para compreender tal processo de ruptura epistemológica, julgamos necessário primeiro analisar 0 campo dos Estudos Culturais e o próprio conceito de diferença.

Nesta perspectiva, na primeira etapa da pesquisa nosso foco privilegiou os Estudos Culturais, buscando identificar alguns dos principais trabalhos da área e, principalmente, as questões que impulsionaram a emergência da disciplina. A partir da leitura dos trabalhos de Stuart Hall (2003), Henry Giroux (2003), Boaventura de Souza Santos (2003), Ana Carolina Escosteguy (2010), Maria Elisa Cevasco (2003), Beatriz Resende (2002) e Eurídice Figueiredo (2010), para citar alguns nomes, foi possível travar contato com as questões formadoras do campo e, principalmente, localizar alguns dos principias pressupostos teóricos.

A pesquisa revelou que Stuart Hall é uma referência teórica incontornável para o nosso estudo. A contribuição de Hall é inegável na formação dos Estudos Culturais e no aprofundamento da reflexão sobre identidade cultural e representação. Para o nosso estudo nos interessou particularmente o debate que o autor realiza sobre o conceito de diferença a partir das contribuições de 
Jacques Derrida (1991) ao propor o neologismo différance. Antes de explicitarmos tal debate e a forma como Hall se apropria do signo derridadiano, se faz necessário apresentarmos, ao menos que de forma sucinta, seu significado. Différance: a discreta intervenção gráfica para a construção do termo, perpetrada pela simples troca da letra e pelo a, ressoa como uma espécie de marca muda, que pode ser lida, escrita, mas não se ouve. Importante observar que différance não é apenas uma palavra ou um conceito, trata-se de um instrumento filosófico que ataca de modo frontal um dos principais sintomas da tradição filosófica ocidental: o fonocentrismo. A apresentação elaborada por Derrida nos permite observar a questão de forma mais objetiva:

Sem dúvida este silêncio piramidal da diferença gráfica entre o e e o a só pode funcionar no interior do sistema de escrita fonética e no interior de uma língua ou de uma gramática historicamente associada à escrita fonética bem como a toda a cultura de que é inseparável. (DERRIDA, 1991, p. 35-6)

A homofonia produzida pela substituição do elemento e de différence pelo a, constituindo o neologismo différance, produz um instrumento de diferenciação duplo: ataca a centralidade da fonética e propõe uma nova ênfase à escrita. A silenciosa subversão do termo diferença em francês - conceito tão caro aos chamados filósofos da diferença, como Nietzsche e Heidegger - produz uma diferença que resulta em uma nova expressão, ou instrumento, que se fixa no jogo entre as formas gráfica e sonora. A deformação perpetrada pela substituição de uma única vogal, que promove uma alteração visual, mas não fonética, objetiva demarcar a diferença entre escrita e fala. Em outras palavras, o termo construído por Derrida apresenta em sua própria estrutura, elaborada na busca pela impossibilidade de semelhança entre o fonético e o gráfico, o desejo de um movimento que produza uma transformação/deformação original. Para além da própria composição do termo e sua consequente deformação e diferença entre a palavra escrita e a inaudível, Derrida apresenta a noção de diferença em oposição ao constante modelo interpretativo que a pensava como resultante e derivada de uma presença prévia.

Antônio Flavio Pierucci, em Ciladas da diferença, define de forma objetiva o resultado do gesto perpetrado pela diferenciação entre linguagem e signo a 
partir da substituição de uma vogal: "Podemos dizer, então, que o a de différance funciona como um ato de diferenciação que produz diferenças. Uma diferença que faz diferença(s)" (Pierucci, 1999, p.146).

Em nosso levantamento bibliográfico foi possível observar que os Estudos Culturais, em especial a partir da produção crítica de Stuart Hall, irão dialogar com o pensamento de Jacques Derrida, elevando o neologismo derridadiano a uma espécie de ferramenta crítica que permite a elaboração de um novo modelo de abordagem dos objetos e de novas concepções do sujeito. O próprio Stuart Hall, em ensaio que examina a construção da noção de multiculturalismo, analisa que seu uso do "conceito" différance é uma certa apropriação, que não intenta rasurar a concepção primeira ofertada por Derrida, mas que o liga a uma leitura do campo cultural enquanto espaço de problematização: "Naturalmente, o que faço aqui é traduzir da filosofia à cultura e expandir o conceito de Derrida sem autorização - embora, espero, não o faça contra o espírito de seu sentido/próposito."(HALL, 2003, p. 92).

A melhor definição para este processo de acomodação do pensamento derridadiano é oferecida pelo próprio Hall, que o nomeia como uma expansão. Nesta perspectiva, não significa que o "conceito" seja alterado ou reelaborado, mas sim que o seu uso é outro, alocado para o exercício crítico de uma outra experiência sensível: a cultura. Para justificar esse uso inusitado, Stuart Hall afirma que "para Derrida, différance é tanto 'marcar diferença' quando 'diferir'. O conceito se funda em estratégias de protelação, suspensão, referência, elisão, desvio, adiamento e reserva" (Idem, Ibidem). O conceito passa, agora, a ser lido enquanto recurso estratégico e não apenas um instrumental teórico. É no intervalo que se cria entre o desejo de marcar a diferença e a localização das diferenças instauradas por outrem - pelo discurso, pelo poder, pelo gênero, pela raça, pela sexualidade, pela classe, pela religião, pela língua, pela deficiência e por tudo o que não se quer diferir ou se deseja alcançar uma igualdade - que se produz um novo ponto de observação do cenário cultural.

No ensaio "Etnicidade: identidade e diferença", fica mais claro o uso que o crítico jamaicano realiza do signo Différance quando o aciona como referência para o exame da relação interdependente entre os conceitos de identidade e 
diferença. Na leitura de Stuart Hall, a lógica da identidade busca uma forma de ancoragem na ideia de autenticidade e se fixa na delimitação de uma diferença. A adoção do signo enquanto recurso crítico para uma abordagem do processo de construção identitária resulta na localização da perspectiva ambivalente própria ao ato discursivo. Dessa forma, após reconhecer as grandes diferenças do discurso do racismo, sempre baseadas em oposições binárias e estanques, como negro e branco, civilizado e primitivo, Norte e Sul, Stuart Hall busca elaborar uma nova concepção de diferença que possa pensar a oposição relacional entre identidade e diferença por um novo prisma. É nesta perspectiva que Différance surge como recurso, o próprio autor observa que

Em Derrida você encontra uma noção de Différance que reconhece a natureza interminável, contínua da construção do significado, mas esta também reconhece que há sempre o jogo da identidade e da diferença e sempre o jogo da diferença sobre a identidade. Você não pode pensar em uma sem a outra. (HALL, 2016, p. 324)

Différance reconhece a natureza interminável, para citar a expressão de Hall, e expande a rigidez da visão agonística que orienta o conceito de diferença. Em suma, se a ideia de diferença necessita da localização do mesmo para se diferir, o pensamento derridadiano nos faz compreender que a diferença deve ser concebida em sua autonomia. Além disso, a interdependência localizada na dualidade existente entre identidade e diferença passa a reverberar um continuo deslocamento, que alimenta o próprio ato discursivo da enunciação. Liv Sovik, na apresentação ao volume Da diáspora, analisa o uso peculiar que Stuart Hall realiza do jogo da diferença, lançando mão do signo "Différance" enquanto recurso discursivo, ao mesmo tempo em que examina o estratégico valor dos discursos de identidade negra diante do racismo:

Hall afirma o valor estratégico dos discursos da identidade negra diante do racismo, com suas múltiplas raízes nos diversos níveis da formação social: político, econômico, social, cultural. Ao mesmo tempo, em um movimento que parece paradoxal, enfoca sempre o jogo da diferença, a différance, a natureza intrinsecamente hibridizada de toda identidade e das identidades diaspóricas em especial. O paradoxo é um lugar que se assume, uma costura de posição e contexto, e não uma essência ou substância a ser examinada. (SOVIK, 2003, p. 15-6) 
A leitura de Liv Sovik nos auxilia no exame da complexa relação entre différance e diferença no pensamento de Hall, nos fazendo observar que não se trata de uma oposição, mas, sim, da acomodação de um novo modelo de interpretação que coaduna as construções identitárias que se desejam fixas e essencialistas ao lado de uma perspectiva híbrida e plural. O resultado desta operação crítica permite observar um aspecto fundamental nas análises da relação entre identidade e diferença: a representação. Afinal, nas palavras do próprio Stuart Hall, "A identidade está dentro do discurso, dentro da representação. Está construída em parte pela representação" (HALL, 2016a, p. 324). Seguindo a trilha aberta pelo autor, somos inclinados a definir que não existe uma identidade fora da representação, sua construção está sempre ligada à linguagem.

Além da reflexão sobre as aproximações dos Estudos Culturais, em especial a produção teórica de Stuart Hall e o pensamento de Jacques Derrida, nossa pesquisa também se ocupou de uma revisão bibliográfica do conceito de diferença. Neste exercício localizamos um número significativo de estudos que acionam a noção de diferença como operador de leitura do cenário cultural contemporâneo. Entre os estudos consultados, consideremos como os mais relevantes os trabalhos de Tomaz Tadeu da Silva, José D'Assunção Barros, Antonio Flavio Pierucci, Boaventura de Sousa Santos e Ernesto Laclau. No entanto, foram as contribuições de Tomaz Tadeu da Silva que se revelaram as mais rentáveis para o desenvolvimento de nossa pesquisa, em especial seu ensaio "A produção social da identidade e da diferença", publicado no livro, Identidade e diferença: a perspectiva dos Estudos Culturais, organizado pelo próprio.

No ensaio o autor analisa a relação interdependente entre os conceitos de identidade e diferença, analisados como peças fundamentais da chamada política de identidade que orienta a agenda política no cenário pós-moderno, em uma perspectiva próxima à elaborada por Stuart Hall. A partir de uma abordagem de acento pós-estruturalista, Tomaz Tadeu da Silva define os conceitos de diferença e identidade enquanto fenômenos ligados a uma prática discursiva, resultado de uma operação linguística ligada ao discurso: 
A identidade e a diferença têm que ser ativamente produzidas. Elas não são criaturas do mundo natural ou do mundo transcendental, mas do mundo cultural e social. Somos nós que as fabricamos, no contexto de relações culturais e sociais. A identidade e a diferença são criações sociais e culturais (Silva, 2014, p.76).

A reflexão do autor é fruto da inspiração pós-estruturalista que norteia o autor, permitindo analisar a dinâmica cultural contemporânea em uma perspectiva crítica, observando os dois conceitos como elementos discursivos fixados em um plano sociocultural de base histórica. Ao se referir à produção de identidade e diferença, o que o autor deseja afirmar é que as identidades e diferenças são construídas através da interação social. Dessa forma toda matriz identitária necessita do conceito e da noção de diferença como movimento inerente a sua formulação.

Em síntese, as identidades são fabricadas por meio da marcação da diferença. Assim, seguindo os passos apontados por Tomaz Tadeu da Silva, podemos aferir que, enquanto frutos de um ato discursivo, identidade e diferença se tornam elementos intercambiáveis, devido ao próprio mecanismo de construção da linguagem. Dessa forma, toda matriz identitária necessita do conceito e da noção de diferença como movimento intrínseco a sua formulação. O movimento de construção identitária resulta de forma direta na demarcação de uma diferenciação. Afinal, quando se delimita o espaço discursivo circunscrito de uma identidade, o gesto secundário e inseparável é a localização da diferença como traço de distinção de uma identidade construída frente às outras. Contudo, nos interessa de modo particular o tratamento que é oferecido à noção de diferença e a forma como esta é tida enquanto uma essência e seus impactos no campo político contemporâneo.

Ao término do levantamento bibliográfico sobre Estudos Culturais e o conceito de diferença, nosso próximo passo foi o investimento em um levantamento de artigos, dissertações, teses e publicações que oferecem um tratamento da surdez e do sujeito surdo à luz do conceito de diferença e a partir do arcabouço teórico formado pelos Estudos Culturais. O trabalho de levantamento bibliográfico foi adotado como metodologia de pesquisa, privilegiando a consulta a periódicos acadêmicos especializados, bancos de 
teses e dissertações, além de publicações voltadas para a temática da educação de surdos e surdez. No trabalho de levantamento bibliográfico realizado no âmbito da pesquisa, privilegiou-se as pesquisas e estudos de intelectuais brasileiros que atuam no campo da educação em interface com os Estudos Culturais. Dessa forma, buscou-se localizar artigos, teses e livros de pesquisadores que desenvolveram estudos sobre a surdez e a educação de surdos em uma perspectiva socioantropológica. Constatamos que os estudos de Carlos Skliar (2013) podem ser tomados como uma importante referência para a construção de um modelo socioantropológico para a compreensão da surdez enquanto uma diferença.

Ao lado do trabalho de Carlos Skliar identificamos também as importantes contribuições de Ana Dorziat (2009), Nídia Regina Limeira de Sá (2006), Karin Strobel (2008), Gladis Perlin (2013) e Marcia Lise Lunardi-Lazzarin (2010), para citar algumas das principais referências da área. Em comum, tais estudos partem da premissa que a surdez se constitui como uma diferença, rompendo com a ideia estanque de deficiência que regia o tratamento da surdez e do sujeito surdo. Nos dizeres de Carlos Skliar, "a surdez constitui uma diferença a ser politicamente reconhecida; a surdez é uma experiência visual; a surdez é uma identidade múltipla ou multifacetada e, finalmente, a surdez está localizada dentro do discurso sobre a deficiência" (SKLIAR, 2013, p.11). Na breve definição do autor está presente um dos principais alicerces teóricos da compreensão da surdez enquanto diferença étnico-linguística, a saber: o reconhecimento político dos sujeitos surdos a partir de suas especificidades linguísticas, culturais e identitárias, conforme observa com grande pertinência César Augusto de Assis Silva, no livro Cultura surda, agentes religiosos e a construção de uma identidade.

Tal investimento teórico tem como objetivo primeiro oferecer uma perspectiva crítica acerca da medicalização da surdez e promover 0 reconhecimento da particularidade linguística e cultural dos surdos. A mudança epistemológica proporcionada pela perspectiva culturalista instaura um modelo de educação bilíngue para os surdos contrastando os outros modelos já existentes: o oralismo e a comunicação total. É importante observar que a defesa 
do modelo de educação bilíngue para surdos emerge em diálogo com as produções acadêmicas da área de linguística sobre a Língua Brasileira de Sinais - Libras e, principalmente, em decorrência do engajamento de associações e federações de surdos em favor da afirmação da Libras como língua natural, movimento este que culminou no dispositivo jurídico recente que reconhece a Libras como uma língua legítima no interior da nação por meio da Lei Federal 10.436/2002. A referida Lei apresenta em seu primeiro artigo a materialidade de um desejo de diferentes atores que atuaram em favor da educação de surdos e na pesquisa e ensino de Libras ao afirmar que:

Art. $1^{\circ}$ É reconhecida como meio legal de comunicação e expressão a Língua Brasileira de Sinais - Libras e outros recursos de expressão a ela associados.

Parágrafo único: Entende-se como Língua Brasileira de Sinais - Libras a forma de comunicação e expressão em que o sistema linguístico de natureza visual-motora, com estrutura gramatical própria, constituem um sistema linguístico de transmissão de ideias e fatos, oriundos de comunidade de pessoas surdas do Brasil (Brasil, 2002, p. 1)

O reconhecimento da Libras como língua natural - conceito linguístico que define que as línguas emergem de forma espontânea de uma comunidade de falantes - oferece à língua de sinais o mesmo estatuto linguístico que qualquer língua oral natural recebe. Afinal, no argumento dos linguistas que se dedicam ao estudo da Libras, esta emergiu de comunidades de sujeitos surdos e são passíveis de análise linguística em diversos planos: sintático, morfológico, fonológico e pragmático. Amparado neste argumento da linguística, o modelo bilíngue de educação de surdos, difundido no Brasil a partir dos estudos de Carlos Skliar (2013), promove um novo tratamento da surdez e coloca em perspectiva a defesa da particularidade linguística do surdo e aciona uma referência culturalista para definir a surdez a partir de uma perspectiva socioantropológico. César Augusto de Assis Silva, em Cultura surda, agentes religiosos e a construção de uma identidade, apresenta de modo objetivo a forma como se constrói o modelo de educação bilíngue de Skliar:

Dada a afirmação da condição de particularidade linguística e cultural dos surdos, Skliar reivindica, de maneira original, a retirada da educação de surdos do âmbito 
da educação especial e deficiência e a colocação dela no debate sobre educação e opressão de minorias (raciais, identitárias, linguísticas e de gênero). (Silva, 2012, p.32)

Ao propor o modelo de educação bilíngue e reivindicar a retirada do tema da surdez - e por conseguinte da educação de surdos - do âmbito da educação especial e do debate sobre deficiência, Carlos Skliar (2013) busca conceituar a surdez enquanto uma diferença em uma perspectiva amparada nos Estudos Culturais. Neste sentido, seguindo o argumento do autor, passamos a compreender a comunidade surda enquanto um grupo minoritário que instaura um elemento de distinção na cultura hegemônica, resultando na construção de uma nova forma de representação do Outro sob o prisma da diferença. Os surdos não são mais sujeitos desviantes de uma norma, de um modelo universal, mas como indicador de outras posturas possíveis. O acionamento deste referencial teórico pretende alcançar a construção de uma leitura da sociedade em uma perspectiva multicultural, na qual todos são iguais respeitando as suas diferenças. No entanto, conforme examina Carlos Skliar,

(...) o conceito de diferença não é utilizado como um termo a mais, dentro de uma continuidade discursiva, em que habitualmente se incluem outros como, por exemplo, "deficiência" ou "diversidade". Esses, no geral, mascaram e neutralizam as possíveis consequências políticas, colocam os outros sob um olhar paternalista e se revelam como estratégias conservadoras para ocultar uma intenção de normalização (Skliar, 2013, p. 5-6).

$\mathrm{Na}$ leitura do pesquisador, a recusa das noções de "deficiência" ou "diversidade", enquanto ferramentas teórico-críticas para a definição da surdez, obedece à compreensão de que tais conceitos apontam para uma certa normalização e mascaram atitudes paternalistas. Nesta perspectiva, a diversidade assume um contorno liberal a partir da presença de conceitos edificantes como da tolerância e do respeito, impedindo a produção de um olhar crítico acerca da dinâmica dos sujeitos e de suas identidades. O próprio Carlos Skliar esclarece que a "diversidade cria um falto consenso, uma ideia de que a normalidade hospeda os diversos, porém mascara normas etnocêntricas e serve para conter a diferença."(Idem, p.13). Por outro lado, o uso do conceito de 
diferença para nomeação da surdez e do sujeito surdo, se coloca em um espaço diametralmente oposto ao discurso paternalista, uma vez que

A diferença como significação política é construída histórica e socialmente; é um processo e um produto de conflitos e movimentos sociais, de resistência às assimetrias de poder e de saber, de uma outra interpretação sobre a alteridade e sobre o significado dos outros no discurso dominante.(Idem, p. 6)

Pensar a surdez enquanto diferença étnico-linguística, conforme exposto aqui em nossa reflexão, não pode significar apenas a adoção de um conceito mais contemporâneo e afeito às modas dos discursos disciplinares. A diferença, principalmente enquanto categoria política, deve ser construída de forma discursiva com o claro intuito de demarcar as contingências do sujeito surdo e da surdez. O uso do conceito de diferença emerge enquanto categoria legitimadora de dois princípios reivindicatórios distintos e complementares: Identidade e Igualdade. Será na produção discursiva dos pares "Identidade e Diferença' e "Igualdade e Diferença" que será edificada a constelação teórica que promove uma definição socioantropológica da surdez. Seja enquanto aparato discursivo interdependente do conceito de identidade ou como categoria desestabilizadora do desejo de normatividade ou homogeneidade, o conceito de diferença rege a concepção culturalista da surdez inaugurada no Brasil por Carlos Skliar (2013).

\section{Referências Bibliográficas}

BARROS, J. D. Igualdade e diferença: construções históricas e imaginárias em torno da desigualdade humana. Petrópolis: Editora Vozes, 2016.

CEVASCO, M. E. Dez lições sobre Estudos Culturais. São Paulo: Boitempo, 2003.

DERRIDA, J. Margens da filosofia. Tradução de Joaquim Torres Costa e Antonio M. Maragalhães. Campinas: Papirus, 1991.

DORZIAT, A. O outro da educação: pensando a surdez com base nos temas identidade/diferença, currículo e inclusão. Petrópolis: Vozes, 2009.

ESCOSTEGUY, A. C. D. Cartografia dos estudos culturais: uma versão latinoamericana. Belo Horizonte: Autêntica, 2010. 
FIGUEIREDO, E. Representações da etnicidade: perspectivas interamericanas de literatura e cultura. Rio de Janeiro: 7 Letras/CNPq, 2010.

GIROUX, H. A. Atos impuros. A prática política dos estudos culturais. Porto Alegre: Artmed Editora, 2003.

HALL, S. Da diáspora: identidades e mediações culturais. Belo Horizonte: UFMG; Brasília: Representação da Unesco no Brasil, 2003.

Etnicidade: identidade e diferença. Tradução de Ana Carolina Cernicchiaro. In: Crítica Cultural. Palhoça, Santa Catarina, v. 11, n. 2, jul/dez., 2016.

LACLAU, E. Emancipação e diferença. Rio de Janeiro: EdUERJ, 2011.

LUNARDI-LAZZARIN, M. L. Os discursos da diferença no contexto das políticas de inclusão: a normalidade no detalhe. In: TREVISAN, A. L.; TOMAZETTI, E. M.; ROSSATO, N. D. (Orgs). Diferença, cultura e educação. Porto Alegre: Sulina, 2010.

2002.

As Identidades Surdas. Revista da FENEIS, Ano IV, n. 14 abr./jun. de

O ser e estar sendo surdos: alteridade, diferença e identidade. Tese (Doutorado em Educação) - Programa de Pós-Graduação em Educação. Universidade Federal do Rio Grande do Sul, Porto Alegre, 2003.

PIERUCCI, A. F. Ciladas da diferença. São Paulo: Editora 34, 1999.

QUADROS, R. M. e KARNOPP, L. B. Língua de sinais brasileira: estudos linguísticos. Porto Alegre: Artmed, 2004.

RESENDE, B. Apontamentos de crítica cultural. Rio de Janeiro: Aeroplano, 2002.

SÁ, N. L. Cultura, poder e educação de surdos. São Paulo: Paulinas, 2006.

SANTOS, B. S. Reconhecer para libertar. Os caminhos do cosmopolitismo multicultural. Rio de Janeiro: Civilização Brasileira, 2003. Coleção Reinventar a emancipação social para novos manifestos - Volume 3.

SILVA, C. A. A. Cultura surda: agentes religiosos e a construção de uma identidade. São Paulo: Terceiro nome, 2012.

SILVA, T. T. (Org.) Identidade e diferença. A perspectiva dos Estudos Culturais. Petrópolis: Editora Vozes, 2014. 
STROBEL, K. As imagens do outro sobre a cultura surda. Florianópolis: Editora da UFSC, 2008.

SKLIAR, Carlos. Os Estudos Surdos em Educação: problematizando a normalidade. In: SKLIAR, Carlos. (Org.). A surdez: um olhar sobre as diferenças. 6. ed. Porto Alegre: Editora Mediação, 2013.

\footnotetext{
Sobre os autores

Paulo Roberto Tonani do Patrocínio

Professor Adjunto do Departamento de Letras-Libras e do Programa de Pós-Graduação em Ciência da Literatura da Faculdade de Letras da UFRJ. É autor dos livros Escritos à margem: a presença de autores de periferia na cena literária brasileira (7Letras/FAPERJ, 2013) e Cidade de lobos: a representação de territórios marginais na obra de Rubens Figueiredo (Ed. UFMG/FAPERJ, 2016) e também co-organizador dos livros de ensaios Modos da margem, figurações da marginalidade na literatura brasileira (Aeroplano, 2015) e Estudos Culturais: legado e apropriações (Ed. Pontes, 2017). Atualmente desenvolve pesquisas sobre o conceito de diferença e representações e autorrepresentações da surdez e de sujeitos surdos.

Ddináh Maria dos Santos

Graduanda do curso de bacharelado de Letras-Libras da Universidade Federal do Rio de Janeiro e bolsista de iniciação científica.
} 